\title{
Scalar, Axial, and Tensor Interactions of Light Nuclei from Lattice QCD
}

\author{
Emmanuel Chang, ${ }^{1}$ Zohreh Davoudi, ${ }^{2,3}$ William Detmold, ${ }^{4,3}$ Arjun S. Gambhir, ${ }^{5,6}$ Kostas Orginos, ${ }^{7,8}$ \\ Martin J. Savage, ${ }^{1,3}$ Phiala E. Shanahan, ${ }^{7,8,3}$ Michael L. Wagman, ${ }^{4,3}$ and Frank Winter ${ }^{8}$
}

(NPLQCD Collaboration)

\author{
${ }_{1}^{1}$ Institute for Nuclear Theory, University of Washington, Seattle, Washington 98195-1550, USA \\ ${ }^{2}$ Department of Physics, University of Maryland, College Park, Maryland 20742, USA \\ ${ }^{3}$ Kavli Institute for Theoretical Physics, University of California, Santa Barbara, California 93106, USA \\ ${ }^{4}$ Center for Theoretical Physics, Massachusetts Institute of Technology, Cambridge, Massachusetts 02139, USA \\ ${ }^{5}$ Lawrence Livermore National Laboratory, Nuclear and Chemical Sciences Division, Livermore, California 94550, USA \\ ${ }^{6}$ Lawrence Berkeley National Laboratory, Nuclear Science Division, Berkeley, California 94720, USA \\ ${ }^{7}$ Department of Physics, College of William and Mary, Williamsburg, Virginia 23187-8795, USA \\ ${ }^{8}$ Jefferson Laboratory, 12000 Jefferson Avenue, Newport News, Virginia 23606, USA
}

(Received 11 January 2018; revised manuscript received 25 February 2018; published 13 April 2018)

\begin{abstract}
Complete flavor decompositions of the matrix elements of the scalar, axial, and tensor currents in the proton, deuteron, diproton, and ${ }^{3} \mathrm{He}$ at $\mathrm{SU}(3)$-symmetric values of the quark masses corresponding to a pion mass $m_{\pi} \sim 806 \mathrm{MeV}$ are determined using lattice quantum chromodynamics. At the physical quark masses, the scalar interactions constrain mean-field models of nuclei and the low-energy interactions of nuclei with potential dark matter candidates. The axial and tensor interactions of nuclei constrain their spin content, integrated transversity, and the quark contributions to their electric dipole moments. External fields are used to directly access the quark-line connected matrix elements of quark bilinear operators, and a combination of stochastic estimation techniques is used to determine the disconnected sea-quark contributions. The calculated matrix elements differ from, and are typically smaller than, naive singlenucleon estimates. Given the particularly large, $\mathcal{O}(10 \%)$, size of nuclear effects in the scalar matrix elements, contributions from correlated multinucleon effects should be quantified in the analysis of dark matter direct-detection experiments using nuclear targets.
\end{abstract}

DOI: 10.1103/PhysRevLett.120.152002

Understanding the spin and flavor structure of nuclei at the level of quarks and gluons is essential to the interpretation of many searches for beyond the Standard Model (BSM) physics. The simplest aspects of the structure of nuclei are revealed through their static responses to external probes. Vector charges of a nucleus are constrained by symmetries and define the number of valence quarks of a given flavor, while matrix elements (MEs) of the axial currents encode the spin carried by quarks and gluons [1-3] and play a central role in weak-interaction processes including single- and double- $\beta$ decay. While difficult to probe experimentally, the (renormalization-scaledependent) scalar and tensor MEs provide important theoretical input for the interpretation of results from dark

Published by the American Physical Society under the terms of the Creative Commons Attribution 4.0 International license. Further distribution of this work must maintain attribution to the author(s) and the published article's title, journal citation, and DOI. Funded by SCOAP ${ }^{3}$. matter direct detection experiments [4] and searches for new physics in precision spectroscopy $[5,6]$. Tensor MEs determine the quark electric dipole moment (EDM) contributions to nuclear EDMs through the dimension-five $C P$ odd operator $\bar{q} \sigma_{\mu \nu} q \tilde{F}^{\mu \nu}$ (where $\tilde{F}^{\mu \nu}=\frac{1}{2} \epsilon^{\mu \nu \rho \sigma} F_{\rho \sigma}$ is the dual of the electromagnetic field strength tensor $F^{\mu \nu}$ and $q$ is the quark field) and are necessary to interpret corresponding searches for BSM $C P$ violation [7-10].

In interpreting intensity-frontier searches for new physics using nuclear targets, it is important to consider multinucleon effects in nuclear MEs. For axial MEs, relevant for Gamow-Teller (GT) transitions, experimental measurements generally differ substantially from naive singlenucleon (NSN) estimates using nuclear ground states with noninteracting nucleons occupying only the lowest shellmodel states [11-15]. Phenomenologically, nuclear shellmodel calculations of $\beta$-decay rates using quenched values of the nucleon axial coupling are known to agree better with experimental values [14-18]. For light nuclei with $A \leq 10$, recent Green function Monte Carlo calculations of GT MEs using chiral currents and potentials [19-23] have shown that 
experimental values of axial MEs can be reproduced by including correlated two-nucleon effects, constrained by experimental observations in few-body systems. In larger nuclei, multibody nuclear effects make such calculations significantly more challenging (see, e.g., Refs. [24,25] for recent progress). For the scalar and tensor currents, chiral effective field theories (EFTs) have also been used to organize multinucleon effects in nuclear MEs [26-42]. In contrast to the axial case, these effects are not well constrained by experiment, but could be determined by matching to $a b$ initio Standard Model calculations of fewbody matrix elements using lattice quantum chromodynamics (lattice QCD). In principle, lattice QCD can be used to calculate the nuclear MEs of interest directly from quark and gluon interactions. While such calculations are extremely challenging, they have now been performed for $A \leq 4$, albeit without fully controlled uncertainties and will be improved and extended to larger nuclei through advances in algorithms and growth of computational resources.

In this Letter, a first-principles lattice QCD study of forward matrix elements of the scalar, axial, and tensor currents, henceforth referred to as "charges," in the nucleon and light nuclei up to atomic number $A=3$ is presented, at unphysical values of the quark masses. These nuclear matrix elements are seen to deviate from the NSN estimates, with particularly large deviations for the scalar current. As many theories of dark matter couple to the Standard Model through scalar exchange, it is important to quantify these potentially large effects in the interpretation of dark matter direct detection experiments.

Lattice QCD methodology.-The numerical calculations presented here are performed using one ensemble of gaugefield configurations generated with a clover-improved fermion action [43] and a Lüscher-Weisz gauge action [44] with $N_{f}=3$ degenerate light-quark flavors. The quark masses are tuned to produce a pion of mass $m_{\pi} \sim 806 \mathrm{MeV}$. The spacetime volume of the ensemble is $L^{3} \times T=32^{3} \times 48$, and the lattice spacing is $a \sim 0.145 \mathrm{fm}$. Further details of the ensemble are given in Ref. [45].

The matrix elements of the scalar, axial, and tensor currents are determined from lattice QCD correlation functions calculated on each gauge-field configuration. These correlation functions separate into two pieces: one in which the quarks interacting with the operator are connected to the hadronic source and sink, and one in which they are not, referred to as quark-line connected and disconnected contributions, respectively. The quark-line connected contributions to the matrix elements are determined using the fixed-order background-field approach introduced in Ref. [46] and discussed in detail for the axial case in Refs. [47-49]. Here, Dirac bilinears $\bar{q} q, \bar{q} \gamma_{3} \gamma_{5} q$, and $\bar{q} \gamma_{1} \gamma_{2} q$ for $q=u, d$ are used to couple to the scalar, axial, and tensor fields, respectively. Correlation functions are constructed with seven different values of the field strengths for proton $p$, deuteron $d$, diproton $p p$, and ${ }^{3} \mathrm{He}$ states. These correlation functions are built from quark propagators originating from a smeared source and having either a smeared (SS) or point (SP) sink [48]. Matrix elements are extracted from the linear responses of the correlation functions to the external fields as detailed in Refs. [46,48,49], using correlated one- and two-state fits. As an illustration, fits determining the connected isoscalar scalar matrix element in ${ }^{3} \mathrm{He}$ are shown in Fig. 1. Fits for all states, as well as details of the statistical sampling, are provided in the Supplemental Material [50]. It is important to note that, at the SU(3)-symmetric value of the quark masses used in this study, all of the states considered are bound ground states with binding energies significantly larger than those in nature [45,51-53] and are spatially compact with respect to the lattice volume. Finite-volume effects in the matrix elements are therefore exponentially small in $\gamma L$, where $\gamma$ is the binding momentum of the system.

Calculation of the quark-line disconnected contributions to each matrix element requires all-to-all propagators, and it is not feasible to compute these objects exactly for the lattice volume used in this Letter. Instead, the requisite traces are estimated stochastically [54-58] using hierarchical probing [59] and singular-value deflation [60,61] (see Refs. $[60,62]$ for complete details). The uncertainties from the stochastic sampling are included in the statistical uncertainties that are presented. The disconnected contributions are subsequently correlated with SS and SP twopoint correlation functions with the relevant quantum numbers to construct the three-point correlation functions. After subtraction of the contributions, in which the current insertions and the two-point functions are uncorrelated, the ratios of the three-point functions to the two-point functions are formed. Finally, the ground-state matrix elements are extracted using correlated two-state fits to the sink and

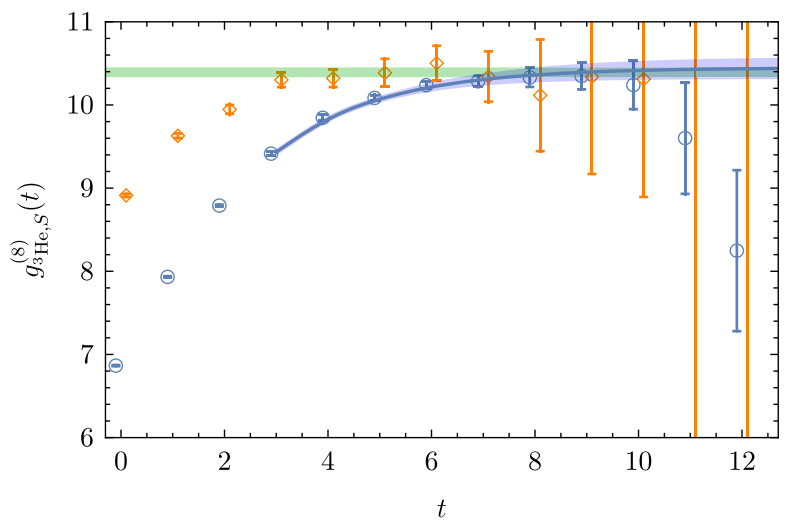

FIG. 1. The bare effective matrix element of the connected isoscalar ${ }^{3} \mathrm{He}$ scalar charge, $g^{(8)} \mathrm{He}, S=\left\langle{ }^{3} \mathrm{He}\left|\bar{q} \Lambda^{(8)} q\right|^{3} \mathrm{He}\right\rangle$, where the blue circles and orange diamonds denote SP and SS results, respectively. The blue band illustrates a correlated two-state fit of the form $\sim A+B e^{-\Delta t}$, to the SP correlation functions, while the green band denotes the final fit result with combined statistical and systematic uncertainties. 


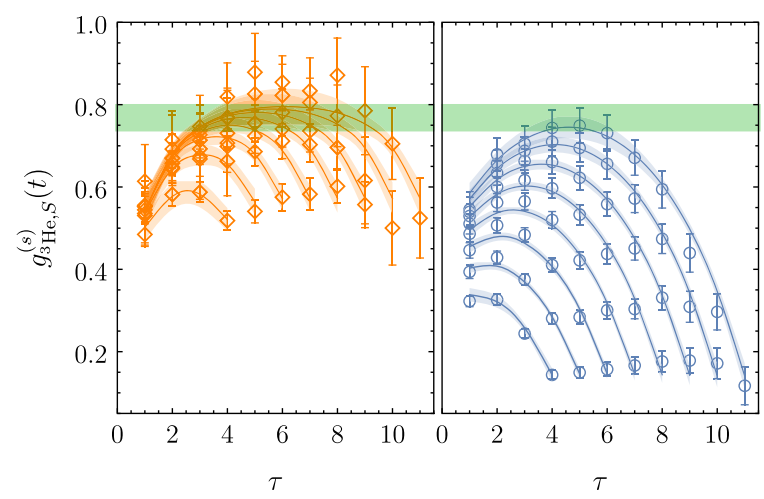

FIG. 2. The strange-quark (disconnected) scalar matrix element in ${ }^{3} \mathrm{He}, g^{3} \mathrm{He}, S=\left\langle{ }^{3} \mathrm{He}|\bar{s} s|{ }^{3} \mathrm{He}\right\rangle$. The left (right) panel shows results obtained using the SS (SP) correlation functions for a range of current insertion times $\tau$ and sink insertion times. The green band corresponds to the extracted matrix element determined as described in the text, and the blue (orange) curves and bands illustrate a correlated two-state fit to the data shown as discussed in the Supplemental Material [50].

operator-insertion time dependence. Bare results for the ${ }^{3} \mathrm{He}$ disconnected scalar matrix element and the corresponding fits are shown in Fig. 2. Further technical details of the statistical sampling of the correlation functions, as well as figures showing the analysis of the other disconnected matrix elements, are presented in the Supplemental Material [50].

Combining the quark-line connected and disconnected contributions to the matrix elements allows a complete flavor decomposition of the scalar, axial, and tensor charges in the light nuclei studied. The bare lattice operators are renormalized using the flavor-nonsinglet renormalization constants $Z_{S}=0.823(16), Z_{A}=0.879(12)$, and $Z_{T}=$ 0.889 (16), determined in Ref. [63] from ensembles with the same action. For the scalar and tensor operators, results are presented in the $\overline{\mathrm{MS}}$ scheme at a renormalization scale of $\mu=2 \mathrm{GeV}$. The isovector charges are free from significant operator mixing, while the isoscalar matrix elements are only determined up to mixings with gluon operators, which are not computed. For the isoscalar scalar and tensor operators, this is a small effect $[64,65]$, while for the isoscalar axial charges, mixing through the flavor-singlet chiral anomaly is potentially significant. Nevertheless, to leading order in the strong coupling, these renormalization factors, mixing, and the renormalization-scale dependence of the scalar and tensor charges, cancel in ratios of nuclear matrix elements to the matrix elements of the same operators in the proton [66]. These ratios, and their differences from the expectations for noninteracting collections of nucleons, encode nuclear effects and are the primary focus of this Letter.

Proton and nuclear charges.-The renormalized scalar, axial, and tensor charges of the proton, deuteron, diproton, and ${ }^{3} \mathrm{He}$ are given in Table I (the bare charges are presented in the Supplemental Material [50]). Results are given in the basis of flavor matrices $\Lambda^{(3)} \equiv \operatorname{diag}(1,-1,0), \quad \Lambda^{(8)} \equiv$ $\operatorname{diag}(1,1,-2)$, and the identity $\Lambda^{(0)} \equiv \operatorname{diag}(1,1,1)$, with the charges labeled as $g_{X}^{(3,8,0)}$, respectively, where $X=S, A$, $T$ indicates the Dirac structure. (For notational convenience, a nonstandard normalization of the flavor matrices is used. These are related to the Gell-Mann matrices $\lambda_{i}$ as $\Lambda^{(3)}=\lambda_{3}$ and $\Lambda^{(8)}=\sqrt{3} \lambda_{8}$.) Since the calculations are performed in the limit of $\mathrm{SU}(3)_{f}$ flavor symmetry, the disconnected contributions cancel in both $g_{X}^{(3)}$ and $g_{X}^{(8)}$. The disconnected contributions (equivalently for the nonstrange hadrons considered here, the strangeness contributions) are defined by the difference $g_{X}^{(\text {disc. })}=g_{X}^{(s)}=\left(g_{X}^{(0)}-g_{X}^{(8)}\right) / 3$. For convenience, these contributions are given separately in Table I.

The ratios of the charges in a nucleus $A$ to those in the proton, $R_{X}^{(f)}(A)=g_{X}^{(f)}(A) / g_{X}^{(f)}(p)$, can be compared with the NSN estimates, defined previously, which are determined entirely by the baryon number, isospin, and spin quantum numbers. Most sources of systematic uncertainty in these calculations, such as lattice spacing and finite volume effects, cancel to a significant extent in these ratios [66]. Figure 3 summarizes the differences $\Delta R_{X}^{(f)}(A)=$ $R_{X}^{(f)}(A)-R_{X}^{(f)}(A)_{\mathrm{NSN}}$, which highlight the effects of

TABLE I. The renormalized scalar, axial, and tensor charges of the proton and light nuclei at a renormalization scale of $\mu=$ $2 \mathrm{GeV}$ in the $\overline{\mathrm{MS}}$ scheme, neglecting mixing with gluonic operators. Specifically, for a nucleus $A, g_{S}^{(i)}=\left\langle A\left|\bar{q} \Lambda^{(i)} q\right| A\right\rangle$, $g_{A}^{(i)}=\left\langle A\left|\bar{q} \gamma_{3} \gamma_{5} \Lambda^{(i)} q\right| A\right\rangle, g_{T}^{(i)}=\left\langle A\left|\bar{q} \sigma_{12} \Lambda^{(i)} q\right| A\right\rangle$, with the flavor structures $\Lambda^{(i)}$ defined in the text. Statistical uncertainties, the systematic uncertainties arising from choices of fit procedure, and the uncertainties of the renormalization constants have been combined in quadrature.

\begin{tabular}{lllcl}
\hline \hline & \multicolumn{1}{c}{$p$} & \multicolumn{1}{c}{$d$} & $p p$ & ${ }^{3} \mathrm{He}$ \\
\hline$g_{S}^{(0)}$ & $3.65(7)$ & $7.20(15)$ & $7.22(15)$ & $10.4(2)$ \\
$g_{S}^{(3)}$ & $0.78(2)$ & & $1.55(4)$ & $0.77(2)$ \\
$g_{S}^{(8)}$ & $2.94(6)$ & $5.84(12)$ & $5.86(12)$ & $8.55(18)$ \\
$g_{S}^{(s)}$ & $0.234(8)$ & $0.45(2)$ & $0.45(2)$ & $0.63(3)$ \\
$g_{A}^{(0)}$ & $0.634(9)$ & $1.26(2)$ & & $0.63(1)$ \\
$g_{A}^{(3)}$ & $1.14(2)$ & & & $1.13(2)$ \\
$g_{A}^{(8)}$ & $0.633(9)$ & $1.25(2)$ & & $0.625(9)$ \\
$g_{A}^{(s)}$ & $0.0002(6)$ & $0.001(1)$ & & $0.003(2)$ \\
$g_{T}^{(0)}$ & $0.684(12)$ & $1.36(2)$ & & $0.678(12)$ \\
$g_{T}^{(3)}$ & $1.12(2)$ & & & $1.12(3)$ \\
$g_{T}^{(8)}$ & $0.684(12)$ & $1.36(2)$ & & $0.676(12)$ \\
$g_{T}^{(s)}$ & $0.00007(13)$ & $0.0002(2)$ & & $0.0004(4)$ \\
\hline \hline
\end{tabular}




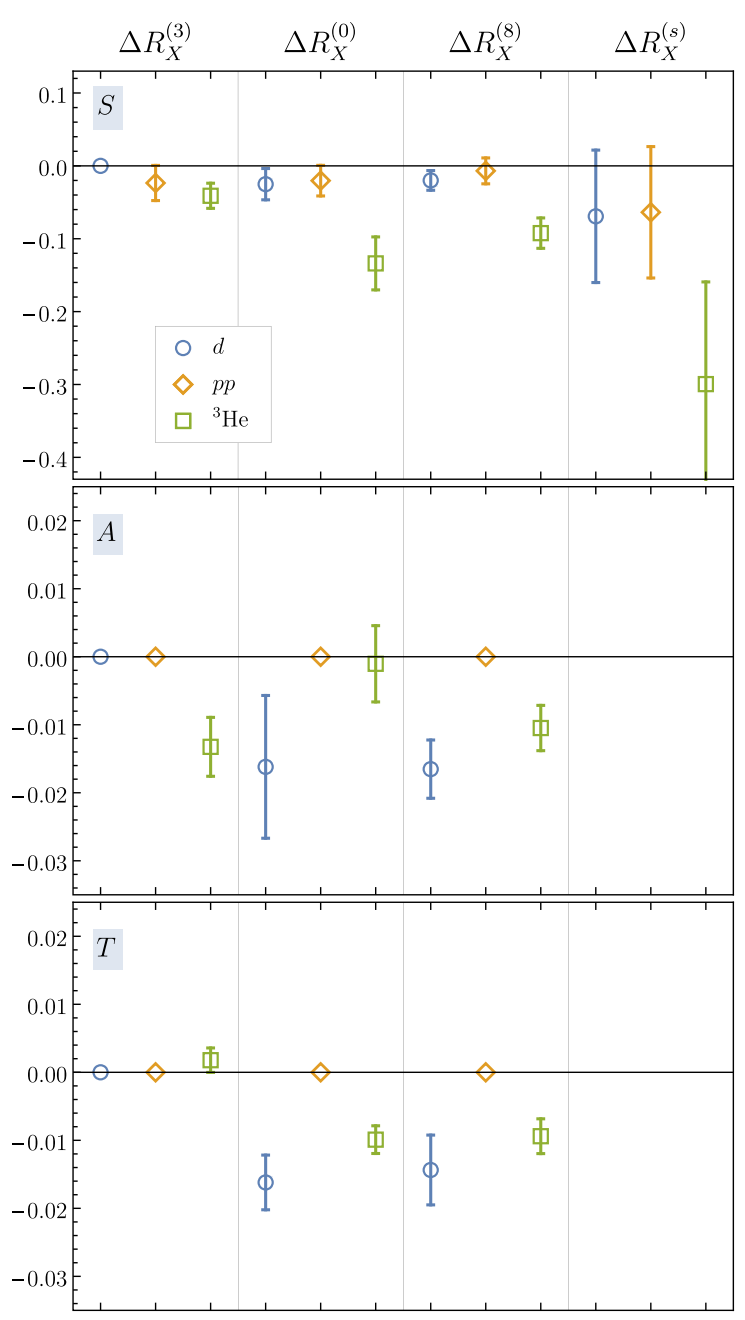

FIG. 3. The calculated values of $\Delta R_{X}^{(f)}$ for the deuteron (circles), diproton (diamonds), and ${ }^{3} \mathrm{He}$ (squares) to those in the proton. The panels display the results obtained for the scalar (top), axial (middle), and tensor (bottom) interactions, and the columns within the panels display results for the different flavor structures of the currents, as indicated at the top of the figure. In each case, the statistical and systematic uncertainties have been combined in quadrature. The points exactly at zero are constrained to vanish by spin and/or isospin symmetry, while ratios are not given for the strange quark axial and tensor charges, as both the numerators and denominators are consistent with zero.

nuclear interactions and correlations on the charges and present a coherent picture of medium effects in light nuclei at $m_{\pi} \sim 806 \mathrm{MeV}$ - the central results of this Letter. Taken as a whole, the results indicate that nuclear effects in the charges are typically at the $\lesssim 2 \%$ level in light nuclei with atomic number $A \leq 3$. The exception to this picture is in the scalar channel, where $\sim 10 \%$ effects are seen. For each type of interaction, nuclear modifications scale approximately with the magnitude of the corresponding charge. While strange quark (equivalently, disconnected) contributions to the nuclear axial and tensor charges are negligible, strange quarks make significant contributions to the scalar charges, as seen for matrix elements of the same operators in the proton in previous studies $[65,67,68]$.

The tensor charges encode the quark EDM contributions to the EDMs of light nuclei and thus set bounds on BSM sources of $C P$ violation [10]. Given that the $C P$ violation in the weak interaction is insufficient to generate the observed matter-antimatter asymmetry of the Universe (assuming exact $C P T$ invariance and baryon-antibaryon symmetry of the initial conditions), many experiments have sought to measure permanent EDMs as evidence for such sources. Even with a successful measurement of a permanent EDM, fully disentangling the sources of $C P$ violation requires multiple observables $[7,69]$, and experiments searching for EDMs of light nuclei are in the planning stages [70-72]. Nuclear effects in the tensor charge have not been previously observed; here they are resolved for the first time and found to be at the few percent level for $A \leq 3$ at these quark masses. Similarly, modification of the axial charge in nuclei is found to be at the $1 \%-2 \%$ level for both the isoscalar and isovector combinations. The isovector ${ }^{3} \mathrm{He}$ charge is consistent with values extracted from measurements of the $\beta$ decay of tritium [73] and is more precise than our previous work [46]. Nuclear effects in the axial charges can test predictions that nuclear modification of the spin-dependent structure function may be significantly different than the modification of the spin-independent structure function [74-76]. The small deviation resolved in this study implies that quarks in nuclei carry a different fraction of the total spin than quarks in free nucleons.

In contrast to the few percent nuclear effects seen in the tensor and axial charges, the scalar charges of light nuclei are suppressed at the $10 \%$ level relative to expectations for noninteracting nucleons. (The sign of these nuclear effects is consistent with the deeper binding of nuclei with increasing quark masses that is found from direct calculations of the binding energies of light nuclei [45].) In phenomenological models of nuclei such as the Walecka model $[77,78]$ and the quark-meson coupling model [79], a mean scalar field in which the nucleons move is an important contribution to the saturation of nuclear matter. The large modifications of the scalar charges found here suggest that models based on similar mechanisms may approximately describe nuclei even at unphysical values of the quark masses. A determination of the scalar polarizabilities through extensions of the calculations presented here (using analogues of the methods discussed in Refs. $[48,49,80])$ would be interesting in this context $[81,82]$.

The scalar charges of nuclei are also important in the interpretation of experimental searches for dark matter [26-31,33-39,41,42]. These charges quantify the contribution of explicit chiral symmetry breaking to nuclear masses $[83,84]$ and define nuclear $\sigma$ terms. The $\sigma$ terms govern the interaction probabilities of many particle dark matter candidates with nuclei in direct detection experiments. The pion and strange $\sigma$ terms for a nucleus $A$ are defined in analogy to 
TABLE II. The $\sigma$ terms of the proton and nuclei at the quark masses used in this Letter. $\sigma_{B ; \pi A}$ and $\sigma_{B ; s A}$ denote the differences between the sigma terms of nuclei and $A$ times the sigma term of the proton [84]. Here $m_{s}=96 \mathrm{MeV}$ has been used, consistent with the physical strange quark mass.

\begin{tabular}{lcccc}
\hline \hline & $p$ & $d$ & $p p$ & ${ }^{3} \mathrm{He}$ \\
\hline$\sigma_{\pi A}(\mathrm{MeV})$ & $327(5)$ & $648(10)$ & $649(10)$ & $942(16)$ \\
$\sigma_{s A}(\mathrm{MeV})$ & $22.5(8)$ & $43.4(19)$ & $43.5(18)$ & $60.7(29)$ \\
$\sigma_{B ; \pi A}(\mathrm{MeV})$ & & $-7(14)$ & $-6(15)$ & $-40(22)$ \\
$\sigma_{B ; s A}(\mathrm{MeV})$ & & $-1.5(2.4)$ & $-1.4(2.4)$ & $-6.7(3.7)$ \\
\hline \hline
\end{tabular}

the nucleon $\sigma$ terms as $\sigma_{\pi A}=m_{l}\langle A|\bar{u} u+\bar{d} d| A\rangle$ and $\sigma_{s A}=m_{s}\langle A|\bar{s} s| A\rangle$, respectively (where $m_{l}$ denotes the average light-quark mass). They can be determined from the scalar charges calculated here and are tabulated in Table II. The results for the light-quark $\sigma$ terms are consistent with, and more precise than, those deduced from numerical lattice QCD calculations for these nuclei using a discretized Feynman-Hellmann approach [84].

Summary.-The results of the lattice QCD calculations presented here reveal percent-level nuclear effects in the axial and tensor charges of light nuclei and $\mathcal{O}(10 \%)$ nuclear effects in the scalar charges, at unphysical values of the quark masses. This is consistent with nucleons being the relevant effective degrees of freedom within these light nuclei, dominating nuclear responses to external probes. Future calculations using additional lattice spacings and volumes, and with the physical values of the quark masses, will determine the scalar, axial, and tensor matrix elements of light nuclei with fully controlled uncertainties. These can then be used to constrain EFT analyses of electroweak interactions with light nuclei and of searches for BSM physics. Specifically, the tensor charges of nuclei are needed for the interpretation $[7,69,85]$ of potential future experimental searches for nuclear EDMs [70-72], and the axial charges control Standard Model predictions for GT transitions and double- $\beta$ decay rates. The scalar charges enter the interpretation of dark matter direct detection experiments and searches for new physics in precision spectroscopy. If the significant nuclear modifications to scalar matrix elements observed in this Letter persist in larger nuclei in nature, such effects will be important for the interpretation of intensity-frontier searches for new physics that employ nuclear targets.

We thank Silas Beane, Martin Hoferichter, Bob McKeown, Assumpta Parreño, Yotam Soreq, Brian Tiburzi, and Nodoka Yamanaka for helpful discussions and comments. We would like to thank Jordy de Vries for emphasizing to us that it may be possible to understand the trends seen in these observables from the nature of the nuclear forces at this pion mass. This research was supported in part by the National Science Foundation under Grant No. NSF PHY11-25915, and Z.D., W. D., M. J.S., P.E. S., and M.L.W. acknowledge the Kavli Institute for Theoretical Physics for hospitality during the development of this work. Calculations were performed using computational resources provided by NERSC (supported by U.S. Department of Energy Award No. DEAC02-05CH11231) and by the USQCD Collaboration. This research used resources of the Oak Ridge Leadership Computing Facility at the Oak Ridge National Laboratory, which is supported by the Office of Science of the U.S. Department of Energy under Award No. DE-AC05-00OR22725. We acknowledge use of the College of William and Mary computing facilities supported by NSF (MRI Grant No. PHY-1626177), the Commonwealth of Virginia Equipment Trust Fund, and the Office of Naval Research. The PRACE Research Infrastructure resources at the Très Grand Centre de Calcul and Barcelona Supercomputing Center were also used. Parts of the calculations used the Chroma software suite [86]. Z. D. was partly supported by the Maryland Center for Fundamental Physics. W. D. was partly supported by U.S. Department of Energy Early Career Research Award DE-SC0010495 and Grant No. DESC0011090. A. G. is supported under the auspices of the U.S. Department of Energy by LLNL under Contract No. DE-AC52-07NA27344. K. O. was partially supported by the U.S. Department of Energy through Award No. DEFG02-04ER41302 and by STFC consolidated Grant No. ST/P000681/1. K. O., P. E. S., and F. W. were partially supported through Contract No. DE-AC05-06OR23177 under which JSA operates the Thomas Jefferson National Accelerator Facility. M. J. S. was supported by DOE Award No. DE-FG02-00ER41132, in part by the USQCD SciDAC project, and by the U.S. Department of Energy through Award No. DE-SC00-10337. M. L. W. was supported by a MIT Pappalardo Fellowship and in part by DOE Award No. DE-SC0011090. F. W. was partially supported through the USQCD Scientific Discovery through Advanced Computing (SciDAC) project funded by the U.S. Department of Energy, Office of Science, Offices of Advanced Scientific Computing Research, Nuclear Physics, and High Energy Physics.

[1] J. Ashman et al. (European Muon Collaboration), Phys. Lett. B 206, 364 (1988).

[2] X.-D. Ji, Phys. Rev. Lett. 78, 610 (1997).

[3] R. L. Jaffe and A. Manohar, Nucl. Phys. B337, 509 (1990).

[4] T. Marrodn Undagoitia and L. Rauch, J. Phys. G 43, 013001 (2016).

[5] C. Delaunay, R. Ozeri, G. Perez, and Y. Soreq, Phys. Rev. D 96, 093001 (2017).

[6] C. Delaunay, C. Frugiuele, E. Fuchs, and Y. Soreq, Phys. Rev. D 96, 115002 (2017).

[7] J. Engel, M. J. Ramsey-Musolf, and U. van Kolck, Prog. Part. Nucl. Phys. 71, 21 (2013). 
[8] N. Yamanaka, Int. J. Mod. Phys. E 26, 1730002 (2017).

[9] N. Yamanaka, B. K. Sahoo, N. Yoshinaga, T. Sato, K. Asahi, and B. P. Das, Eur. Phys. J. A 53, 54 (2017).

[10] T. Chupp, P. Fierlinger, M. Ramsey-Musolf, and J. Singh, arXiv: 1710.02504.

[11] B. Buck and S. M. Perez, Phys. Rev. Lett. 50, 1975 (1983).

[12] D. Krofcheck, E. Sugarbaker, J. Rapaport, D. Wang, R. Byrd, C. Foster, C. Goodman, I. V. Heerden, T. Taddeucci, J. N. Bahcall et al., Phys. Rev. Lett. 55, 1051 (1985).

[13] W.-T. Chou, E. K. Warburton, and B. A. Brown, Phys. Rev. C 47, 163 (1993).

[14] B. A. Brown, W. Chung, and B. H. Wildenthal, Phys. Rev. Lett. 40, 1631 (1978).

[15] B. H. Wildenthal, M. S. Curtin, and B. A. Brown, Phys. Rev. C 28, 1343 (1983).

[16] G. Martinez-Pinedo, A. Poves, E. Caurier, and A. P. Zuker, Phys. Rev. C 53, R2602 (1996).

[17] V. Kumar, P. C. Srivastava, and H. Li, J. Phys. G 43, 105104 (2016).

[18] F. F. Deppisch and J. Suhonen, Phys. Rev. C 94, 055501 (2016).

[19] R. Schiavilla et al., Phys. Rev. C 58, 1263 (1998).

[20] H. Krebs, E. Epelbaum, and U. G. Meiner, Ann. Phys. (Amsterdam) 378, 317 (2017).

[21] A. Baroni, L. Girlanda, A. Kievsky, L. E. Marcucci, R. Schiavilla, and M. Viviani, Phys. Rev. C 94, 024003 (2016); Phys. Rev. C 95, 059902(E) (2017).

[22] H. De-Leon, L. Platter, and D. Gazit, arXiv:1611.10004.

[23] S. Pastore, A. Baroni, J. Carlson, S. Gandolfi, S. C. Pieper, R. Schiavilla, and R. B. Wiringa, Phys. Rev. C 97, 022501 (2018).

[24] T. Shafer, J. Engel, C. Fröhlich, G. C. McLaughlin, M. Mumpower, and R. Surman, Phys. Rev. C 94, 055802 (2016).

[25] E. A. C. Prez, J. Menndez, and A. Schwenk, arXiv:1708 .06140 .

[26] J. Engel, S. Pittel, and P. Vogel, Int. J. Mod. Phys. E 01, 1 (1992).

[27] G. Prezeau, A. Kurylov, M. Kamionkowski, and P. Vogel, Phys. Rev. Lett. 91, 231301 (2003).

[28] J. Ellis, K. A. Olive, and C. Savage, Phys. Rev. D 77, 065026 (2008).

[29] J. Ellis, K. A. Olive, and P. Sandick, New J. Phys. 11, 105015 (2009).

[30] J. Giedt, A. W. Thomas, and R. D. Young, Phys. Rev. Lett. 103, 201802 (2009).

[31] R. J. Hill and M. P. Solon, Phys. Lett. B 707, 539 (2012).

[32] J. Menendez, D. Gazit, and A. Schwenk, Phys. Rev. Lett. 107, 062501 (2011).

[33] J. Ellis and K. A. Olive, Eur. Phys. J. C 72, 2005 (2012).

[34] S. J. Underwood, J. Giedt, A. W. Thomas, and R. D. Young, Phys. Rev. D 86, 035009 (2012).

[35] C. Korber, A. Nogga, and J. de Vries, Phys. Rev. C 96, 035805 (2017).

[36] V. Cirigliano, M. L. Graesser, and G. Ovanesyan, J. High Energy Phys. 10 (2012) 025.

[37] A. L. Fitzpatrick, W. Haxton, E. Katz, N. Lubbers, and Y. Xu, J. Cosmol. Astropart. Phys. 02 (2013) 004.

[38] P. Klos, J. Menendez, D. Gazit, and A. Schwenk, Phys. Rev. D 88, 083516 (2013); Phys. Rev. D 89, 029901(E) (2014).
[39] M. Hoferichter, P. Klos, and A. Schwenk, Phys. Lett. B 746, 410 (2015).

[40] M. Hoferichter, P. Klos, J. Menendez, and A. Schwenk, Phys. Rev. D 94, 063505 (2016).

[41] F. Bishara, J. Brod, B. Grinstein, and J. Zupan, J. Cosmol. Astropart. Phys. 02 (2017) 009.

[42] M. Hoferichter, P. Klos, J. Menendez, and A. Schwenk, Phys. Rev. Lett. 119, 181803 (2017).

[43] B. Sheikholeslami and R. Wohlert, Nucl. Phys. B259, 572 (1985).

[44] M. Lüscher and P. Weisz, Commun. Math. Phys. 97, 59 (1985).

[45] S. R. Beane, E. Chang, S. D. Cohen, W. Detmold, H. W. Lin, T. C. Luu, K. Orginos, A. Parreno, M. J. Savage, and A. Walker-Loud (NPLQCD Collaboration), Phys. Rev. D 87, 034506 (2013).

[46] M. J. Savage, P. E. Shanahan, B. C. Tiburzi, M. L. Wagman, F. Winter, S. R. Beane, E. Chang, Z. Davoudi, W. Detmold, and K. Orginos, Phys. Rev. Lett. 119, 062002 (2017).

[47] C. Bouchard, C. C. Chang, T. Kurth, K. Orginos, and A. Walker-Loud, Phys. Rev. D 96, 014504 (2017).

[48] B. C. Tiburzi, M. L. Wagman, F. Winter, E. Chang, Z. Davoudi, W. Detmold, K. Orginos, M. J. Savage, and P. E. Shanahan, Phys. Rev. D 96, 054505 (2017).

[49] P. E. Shanahan, B. C. Tiburzi, M. L. Wagman, F. Winter, E. Chang, Z. Davoudi, W. Detmold, K. Orginos, and M. J. Savage, Phys. Rev. Lett. 119, 062003 (2017).

[50] See Supplemental Material at http://link.aps.org/ supplemental/10.1103/PhysRevLett.120.152002 for technical details of the LQCD computation and numerical analysis, as well as the effective ratio plots that obtain all the charges along with their bare values.

[51] K. Orginos, A. Parreno, M. J. Savage, S. R. Beane, E. Chang, and W. Detmold, Phys. Rev. D 92, 114512 (2015).

[52] M. L. Wagman, F. Winter, E. Chang, Z. Davoudi, W. Detmold, K. Orginos, M. J. Savage, and P. E. Shanahan, Phys. Rev. D 96, 114510 (2017).

[53] S. R. Beane et al., arXiv:1705.09239.

[54] M. F. Hutchinson, J. Commun. Stat. Simul. 19, 433 (1990).

[55] S.-J. Dong and K.-F. Liu, Phys. Lett. B 328, 130 (1994).

[56] H. Neff, N. Eicker, T. Lippert, J. W. Negele, and K. Schilling, Phys. Rev. D 64, 114509 (2001).

[57] J. Foley, K. J. Juge, A. O'Cais, M. Peardon, S. Ryan, and J.-I. Skullerud, Comput. Phys. Commun. 172, 145 (2005).

[58] R. Babich, R. Brower, M. Clark, G. Fleming, J. Osborn, and C. Rebbi, Proc. Sci., LATTICE2007 (2007) 139 [arXiv: 0710.5536].

[59] A. Stathopoulos, J. Laeuchli, and K. Orginos, arXiv: 1302.4018

[60] A. S. Gambhir, A. Stathopoulos, and K. Orginos, SIAM J. Sci. Comput. 39, A532 (2017).

[61] A. S. Gambhir, A. Stathopoulos, K. Orginos, B. Yoon, R. Gupta, and S. Syritsyn, Proc. Sci., LATTICE2016 (2016) 265 [arXiv:1611.01193].

[62] Arjun Gambhir, Ph.D. Thesis, College of William and Mary, 2017.

[63] B. Yoon et al., Phys. Rev. D 95, 074508 (2017).

[64] C. Alexandrou, M. Constantinou, K. Hadjiyiannakou, K. Jansen, H. Panagopoulos, and C. Wiese, Phys. Rev. D 96, 054503 (2017). 
[65] C. Alexandrou et al., Phys. Rev. D 95, 114514 (2017); Phys. Rev. D 96, 099906(E) (2017).

[66] F. Winter, W. Detmold, A. S. Gambhir, K. Orginos, M. J. Savage, P. E. Shanahan, and M. L. Wagman, Phys. Rev. D 96, 094512 (2017).

[67] J. Green, S. Meinel, M. Engelhardt, S. Krieg, J. Laeuchli, J. Negele, K. Orginos, A. Pochinsky, and S. Syritsyn, Phys. Rev. D 92, 031501 (2015).

[68] T. Bhattacharya, V. Cirigliano, S. D. Cohen, R. Gupta, A. Joseph, H.-W. Lin, and B. Yoon (PNDME Collaboration), Phys. Rev. D 92, 094511 (2015).

[69] E. Mereghetti and U. van Kolck, Annu. Rev. Nucl. Part. Sci. 65, 215 (2015).

[70] Y. K. Semertzidis et al. (EDM Collaboration), AIP Conf. Proc. 698, 200 (2004).

[71] Y. K. Semertzidis (Storage Ring EDM Collaboration), in Particles and Fields. Proceedings, Meeting of the Division of the American Physical Society, DPF 2011, Providence, RI, 2011, https://inspirehep.net/record/940136/files/arXiv: 1110.3378.pdf (unpublished).

[72] J. Pretz (JEDI Collaboration), Hyperfine Interact. 214, 111 (2013).

[73] J. C. Hardy and I. S. Towner, Phys. Rev. C 91, 025501 (2015).
[74] J.-W. Chen and W. Detmold, Phys. Lett. B 625, 165 (2005).

[75] I. C. Cloet, W. Bentz, and A. W. Thomas, Phys. Rev. Lett. 95, 052302 (2005).

[76] J. R. Smith and G. A. Miller, Phys. Rev. C 72, 022203 (2005).

[77] J. D. Walecka, Ann. Phys. (N.Y.) 83, 491 (1974).

[78] B. D. Serot and J. D. Walecka, Adv. Nucl. Phys. 16, 1 (1986).

[79] K. Saito and A. W. Thomas, Phys. Lett. B 327, 9 (1994).

[80] E. Chang, W. Detmold, K. Orginos, A. Parreño, M. J. Savage, B. C. Tiburzi, and S. R. Beane (NPLQCD Collaboration), Phys. Rev. D 92, 114502 (2015).

[81] P. A. M. Guichon, Phys. Lett. B 200, 235 (1988).

[82] J. R. Stone, P. A. M. Guichon, P. G. Reinhard, and A. W. Thomas, Phys. Rev. Lett. 116, 092501 (2016).

[83] M. C. Birse, J. Phys. G 20, 1537 (1994).

[84] S. R. Beane, S. D. Cohen, W. Detmold, H. W. Lin, and M. J. Savage, Phys. Rev. D 89, 074505 (2014).

[85] A. Wirzba, J. Bsaisou, and A. Nogga, Int. J. Mod. Phys. E 26, 1740031 (2017).

[86] R. G. Edwards and B. Joo (SciDAC Collaboration), Nucl. Phys. B, Proc. Suppl. 140, 832 (2005). 